EDITORIAL

\title{
ETHICAL RESPONSIBILITIES OF THE AUTHORS
}

According to the proposed international standards, the respective separate section should be placed in all issues after the main text and before the list of references of any paper. It should include the following points.

\section{Acknowledgements.}

If the study was carried out with additional financial and/or organizational support, all sources of the latter should be mentioned. Research grants from funding agencies should be indicated; separate subjects who received these grants (if they are among the authors) should be indicated personally; the research funder and the grant number should be mentioned. If the grant was received by a separate co-author or some co-authors, the respective information should be presented.

If necessary or desirable, the gratitude of the authors to the persons and/or organizations providing valuable assistance in the performance of the study or its separate stages should be expressed.

Then, the ethical statement that all research procedures were in accordance with international ethical standards should be given.

In the case of experiments on animals, the compliance of the study to the statements of the Council Directive regarding the protection of animals used for experimental and other scientific purposes (86/609/EEC, 1986, Strasbourg) and respective regulations of the national and local Ethics Committees should be mentioned. For the studies on non-animal objects (e.g., cell cultures), it is necessary to indicate the source of the respective material.

In the case of tests on or examinations of humans, it should be mentioned that the study was carried out in accordance with the Helsinki Declaration (1975, revised in 1996-2013). The statement that preliminary written informed consent was obtained from the subjects involved in the tests is absolutely necessary. In the case where children or patients were involved in the study, this consent should be given by responsible persons (parents, guardians, attending doctors, etc.)

For certain types of the papers (e.g., reviews, computer modeling, etc.), the sentence of such a type should be added: "This article does not contain any studies with human participants or animals, and formal consent is not required".

The statement of potential conflicts of interests should include indications on the absence of any conflict, with regard to both commercial and financial relations, to organizations or persons that could be involved in some way in the study, and to interrelations between co-authors of the paper (if the latter was written by a group of researchers). The names of all co-authors of the paper should be mentioned in this paragraph. This statement means that the authors assume full responsibility for a possible conflict of interest. 